\title{
ALMOST EVEN-CLIFFORD HERMITIAN MANIFOLDS WITH A LARGE AUTOMORPHISM GROUP
}

\author{
GERARDO ARIZMENDI, RAFAEL HERRERA, AND NOEMI SANTANA
}

(Communicated by Lei $\mathrm{Ni}$ )

\begin{abstract}
We study manifolds endowed with an (almost) even Clifford (hermitian) structure and admitting a large automorphism group. We classify them when they are simply connected and the dimension of the automorphism group is maximal, and also prove a gap theorem for the dimension of the automorphism group.
\end{abstract}

\section{INTRODUCTION}

Recently, there has been some interest in manifolds admitting so-called even Clifford structures [9, 10. Here, we study such manifolds when they admit a large automorphism group. This type of problem has been studied on Riemannian manifolds [13, 14, almost hermitian manifolds [12], and almost quaternion-hermitian manifolds [1].

It is a classical result [6] that the maximal dimension of the isometry group of a connected $n$-dimensional Riemannian manifold is $\frac{1}{2} n(n+1)$. If the dimension is maximal, the manifold is isometric to either Euclidean space $\mathbb{R}^{n}$, or the sphere $S^{n}$, or projective space $\mathbb{R P}^{n}$, or a (simply connected) hyperbolic space. Furthermore, in 13 it was shown that the isometry group contains no $m$-dimensional closed subgroup where

$$
\frac{1}{2} n(n-1)+1<m<\frac{1}{2} n(n+1) .
$$

In [12, it was shown that the automorphism group of a connected $2 n$-dimensional almost-hermitian manifold has dimension at most $n(n+2)$. If the dimension of the automorphism is maximal, the manifold is isometric to either complex Euclidean space $\mathbb{C}^{n}$, or an open ball with Kähler structure with negative constant holomorphic sectional curvature, or complex projective space $\mathbb{C P}^{n}$ (cf. [4]).

In [11, it was shown that the automorphism group of a connected $4 n$-dimensional almost quaternion-hermitian manifold has dimension at most $2 n^{2}+5 n+3$. If the dimension of the automorphism group is between $2 n^{2}+5 n$ and $2 n^{2}+5 n+3$, the manifold is isometric to either quaternionic Euclidean space $\mathbb{H}^{n}$, or quaternionic projective space $\mathbb{H} \mathbb{P}^{n}$, or quaternionic hyperbolic space.

In this paper, we will prove the analogous theorems for almost even-Clifford hermitian manifolds of rank $r \geq 3$. Our terminology differs from that of 9 , 10] since we have added the words "almost" and "hermitian" because, in principle,

Received by the editors November 4, 2015.

2010 Mathematics Subject Classification. Primary 53C10, 53C15, 53C26, 53C35.

The first author was partially supported by a CONACyT scholarship. The second and third authors were partially supported by grants from CONACyT and LAISLA (CONACyT-CNRS). 
there is no integrability condition on the structure and the compatibility with a Riemannian metric is an extra condition. We shall explore integrability conditions in the style of Gray [3] in a future paper.

The paper is organized as follows. In Section 2 we recall some preliminaries on Clifford algebras and representations, and almost even-Clifford hermitian manifolds. In Section 3 we give an upper bound in the dimension of the automorphism group (Proposition 3.1), determine the spaces whose automorphism group has dimension equal to this bound (Theorem [3.1), and prove a gap in the dimension of the automorphism group (Proposition 3.2 ).

\section{Preliminaries}

In this section we recall material that can also be consulted in [2,7]. Let $C l_{r}$ denote the Clifford algebra generated by all the products of the orthonormal vectors $e_{1}, e_{2}, \ldots, e_{r} \in \mathbb{R}^{r}$ subject to the relations

$$
e_{j} e_{k}+e_{k} e_{j}=-2 \delta_{j k}, \quad \text { for } 1 \leq j, k \leq r .
$$

The even Clifford subalgebra $C l_{r}^{0}$ is defined as the invariant $(+1)$-subspace of the involution of $C l_{r}$ induced by the map $-\operatorname{Id}_{\mathbb{R}^{r}}$. The Spin group $\operatorname{Spin}(r) \subset C l_{r}$ is the subset

$$
\operatorname{Spin}(r)=\left\{x_{1} x_{2} \cdots x_{2 l-1} x_{2 l}\left|x_{j} \in \mathbb{R}^{r},\right| x_{j} \mid=1, l \in \mathbb{N}\right\},
$$

endowed with the product of the Clifford algebra. The Lie algebra of $\operatorname{Spin}(r)$ is

$$
\mathfrak{s p i n}(r)=\operatorname{span}\left\{e_{i} e_{j} \mid 1 \leq i<j \leq r\right\} .
$$

Now, we summarize some results about real representations of $C l_{r}^{0}$ in Table 1 (cf. 7]). Here $d_{r}$ denotes the dimension of an irreducible representation of $C l_{r}^{0}$ and $v_{r}$ the number of distinct irreducible representations. Let $\tilde{\Delta}_{r}$ denote the irreducible representation of $C l_{r}^{0}$ for $r \not \equiv 0(\bmod 4)$ and $\tilde{\Delta}_{r}^{ \pm}$denote the irreducible representations for $r \equiv 0(\bmod 4)$.

TABLE 1

\begin{tabular}{|c|c|c|c|c|}
\hline$r(\bmod 8)$ & $d_{r}$ & $C l_{r}^{0}$ & $\tilde{\Delta}_{r} / \tilde{\Delta}_{r}^{ \pm} \cong \mathbb{R}^{d_{r}}$ & $v_{r}$ \\
\hline 1 & $2^{\left\lfloor\frac{r}{2}\right\rfloor}$ & $\mathbb{R}\left(d_{r}\right)$ & $\mathbb{R}^{d_{r}}$ & 1 \\
\hline 2 & $2^{\frac{r}{2}}$ & $\mathbb{C}\left(d_{r} / 2\right)$ & $\mathbb{C}^{d_{r} / 2}$ & 1 \\
\hline 3 & $2^{\left\lfloor\frac{r}{2}\right\rfloor+1}$ & $\mathbb{H}\left(d_{r} / 4\right)$ & $\mathbb{H}^{d_{r} / 4}$ & 1 \\
\hline 4 & $2^{\frac{r}{2}}$ & $\mathbb{H}\left(d_{r} / 4\right) \oplus \mathbb{H}\left(d_{r} / 4\right)$ & $\mathbb{H}^{d_{r} / 4}$ & 2 \\
\hline 5 & $2^{\left\lfloor\frac{r}{2}\right\rfloor+1}$ & $\mathbb{H}\left(d_{r} / 4\right)$ & $\mathbb{H}^{d_{r} / 4}$ & 1 \\
\hline 6 & $2^{\frac{r}{2}}$ & $\mathbb{C}\left(d_{r} / 2\right)$ & $\mathbb{C}^{d_{r} / 2}$ & 1 \\
\hline 7 & $2^{\left\lfloor\frac{r}{2}\right\rfloor}$ & $\mathbb{R}\left(d_{r}\right)$ & $\mathbb{R}^{d_{r}}$ & 1 \\
\hline 8 & $2^{\frac{r}{2}-1}$ & $\mathbb{R}\left(d_{r}\right) \oplus \mathbb{R}\left(d_{r}\right)$ & $\mathbb{R}^{d_{r}}$ & 2 \\
\hline
\end{tabular}

Note that the representations are complex for $r \equiv 2,6(\bmod 8)$ and quaternionic for $r \equiv 3,4,5(\bmod 8)$. 


\section{Almost even-Clifford hermitian structures.}

Definition 2.1. A linear even-Clifford hermitian structure of rank $r$ on $\mathbb{R}^{N}, N \in \mathbb{N}$, is a representation

$$
C l_{r}^{0} \longrightarrow \operatorname{End}\left(\mathbb{R}^{N}\right)
$$

such that each bivector $e_{i} e_{j}, 1 \leq i<j \leq r$, is mapped to an antisymmetric endomorphism $J_{i j}$ satisfying

$$
J_{i j}^{2}=-\operatorname{Id}_{\mathbb{R}^{N}} .
$$

Notice that the subalgebra $\mathfrak{s p i n}(r)$ is mapped injectively into the skew-symmetric endomorphisms $\operatorname{End}^{-}\left(\mathbb{R}^{N}\right)$.

First, let us assume $r \not \equiv 0(\bmod 4), r>1$. In this case, $\mathbb{R}^{N}$ decomposes into a sum of irreducible representations of $C l_{r}^{0}$. Since this algebra is simple, such irreducible representations can only be trivial or copies of the standard representation $\tilde{\Delta}_{r}$ of $C l_{r}^{0}$ (cf. [7]). Due to (1), there are no trivial summands in such a decomposition so that

$$
\mathbb{R}^{N}=\tilde{\Delta}_{r} \otimes_{\mathbb{R}} \mathbb{R}^{m}
$$

for some $m \in \mathbb{N}$. Thus, we see that $\mathfrak{s p i n}(r)$ has an isomorphic image

$$
\widehat{\mathfrak{s p i n}(r)}:=\mathfrak{s p i n}(r) \otimes\left\{\operatorname{Id}_{m \times m}\right\} \subset \mathfrak{s o}\left(d_{r} m\right) .
$$

Secondly, let us assume $r \equiv 0(\bmod 4)$. Recall that if $\hat{\Delta}_{r}$ is the irreducible representation of $C l_{r}$, then by restricting this representation to $C l_{r}^{0}$ it splits as the sum of two inequivalent irreducible representations

$$
\hat{\Delta}_{r}=\tilde{\Delta}_{r}^{+} \oplus \tilde{\Delta}_{r}^{-} .
$$

Since $\mathbb{R}^{N}$ is a representation of $C l_{r}^{0}$ satisfying (1), there are no trivial summands in such a decomposition so that

$$
\mathbb{R}^{N}=\tilde{\Delta}_{r}^{+} \otimes \mathbb{R}^{m_{1}} \oplus \tilde{\Delta}_{r}^{-} \otimes \mathbb{R}^{m_{2}},
$$

for some $m_{1}, m_{2} \in \mathbb{N}$. By restricting this representation to $\mathfrak{s p i n}(r) \subset C l_{r}^{0}$, consider

$$
\begin{aligned}
\widehat{\operatorname{spin}(r)} & :=\left\{g^{+} \otimes \operatorname{Id}_{m_{1} \times m_{1}} \oplus g^{-} \otimes \operatorname{Id}_{m_{2} \times m_{2}}\left|g \in \operatorname{Spin}(r), g^{+}=g\right|_{\tilde{\Delta}_{r}^{+}}, g^{-}=\left.g\right|_{\tilde{\Delta}_{r}^{-}}\right\} \\
& \subset \mathfrak{s o}\left(d_{r} m_{1}+d_{r} m_{2}\right),
\end{aligned}
$$

where $\mathfrak{s p i n}(r)^{ \pm}$are the images of $\mathfrak{s p i n}(r)$ in $\operatorname{End}\left(\tilde{\Delta}_{r}^{ \pm}\right)$respectively.

Definition 2.2. $\quad$ - A rank $r$ almost even-Clifford hermitian structure, $r \geq$ 2, on a Riemannian manifold $M$ is a smoothly varying choice of linear even-Clifford hermitian structure on each tangent space of $M$. Let $Q \subset$ $\operatorname{End}^{-}(T M)$ denote the subbundle with fiber $\mathfrak{s p i n}(r)$.

- A Riemannian manifold carrying such a structure will be called an almost even-Clifford hermitian manifold.

- An almost even-Clifford hermitian structure on a Riemannian manifold $M$ is called parallel if the bundle $Q$ is parallel with respect to the Levi-Civita connection on $M$.

Notice that the definition of a (parallel) even-Clifford structure in 9 implies the one we have just given. 


\section{Automorphism group}

In this section we derive an upper bound on the dimension of the automorphism group of an almost even-Clifford hermitian manifold and classify the manifolds whose automorphism group's dimension attains such an upper bound.

The automorphism group of an almost even-Clifford hermitian manifold $M$, denoted by $\operatorname{Aut}(M)$, is the (sub)group of isometries which preserve the almost evenClifford hermitian structure. A vector field $X$ on $M$ is an infinitesimal automorphism if it is a Killing vector field that preserves the structure, i.e. locally

$$
\mathcal{L}_{X} J_{i j}=\sum_{k<l} \alpha_{k l}^{(i j)} J_{k l}
$$

for some (local) functions $\alpha_{k l}^{(i j)}$, where $\mathcal{L}_{X}$ denotes the Lie derivative in the direction of $X$. These vector fields form the Lie algebra aut $(M)$ of $\operatorname{Aut}(M)$.

3.1. Upper bound. Let $X$ be an infinitesimal automorphism of $M$. Consider

$$
\mathcal{L}_{X}\left(J_{i j}(Y)\right)=\left(\mathcal{L}_{X} J_{i j}\right)(Y)+J_{i j}\left(\mathcal{L}_{X} Y\right),
$$

i.e.

$$
\nabla_{X}\left(J_{i j}(Y)\right)-\nabla_{J_{i j}(Y)} X=\sum_{k<l} \alpha_{k l}^{(i j)} J_{k l}(Y)+J_{i j}\left(\nabla_{X} Y-\nabla_{Y} X\right)
$$

Now suppose we are calculating at a point $p$ where $X_{p}=0$, so that

$$
-\nabla_{J_{i j}(Y)} X=\sum_{k<l} \alpha_{k l}^{(i j)} J_{k l}(Y)-J_{i j}\left(\nabla_{Y} X\right),
$$

i.e.

$$
\left[J_{i j}, \nabla X\right](Y)=\sum_{k<l} \alpha_{k l}^{(i j)} J_{k l}(Y) .
$$

Hence, $(\nabla X)_{p}$ is a skew-symmetric endomorphism such that

$$
\left[J_{i j}, \nabla X\right]=\sum_{k<l} \alpha_{k l}^{(i j)} J_{k l}
$$

i.e. $(\nabla X)_{p}$ belongs to the normalizer of $\widehat{\mathfrak{s p i n}(r)}=\operatorname{span}\left(J_{i j}\right)$ in $\operatorname{End}^{-}\left(T_{p} M\right)=$ $\mathfrak{s o}\left(T_{p} M\right)$. Such normalizers have been calculated in [1] and we list them for $r \geq 3$.

TABLE 2

\begin{tabular}{|c|c|c|c|}
\hline$r(\bmod 8)$ & $N$ & $N_{\mathfrak{s o}(N)}(\widehat{\mathfrak{s p i n}(r)})$ & $\left.C_{\mathfrak{s o}(N)} \widehat{(\mathfrak{s p i n}(r)}\right)$ \\
\hline 0 & $d_{r}\left(m_{1}+m_{2}\right)$ & $\mathfrak{s o}\left(m_{1}\right) \oplus \mathfrak{s o}\left(m_{2}\right) \oplus \mathfrak{s p i n}(r)$ & $\mathfrak{s o}\left(m_{1}\right) \oplus \mathfrak{s o}\left(m_{2}\right)$ \\
\hline 1 & $d_{r} m$ & $\mathfrak{s o}(m) \oplus \mathfrak{s p i n}(r)$ & $\mathfrak{s o}(m)$ \\
\hline 2 & $d_{r} m$ & $\mathfrak{u}(m) \oplus \mathfrak{s p i n}(r)$ & $\mathfrak{u}(m)$ \\
\hline 3 & $d_{r} m$ & $\mathfrak{s p}(m) \oplus \mathfrak{s p i n}(r)$ & $\mathfrak{s p}(m)$ \\
\hline 4 & $d_{r}\left(m_{1}+m_{2}\right)$ & $\mathfrak{s p}\left(m_{1}\right) \oplus \mathfrak{s p}\left(m_{2}\right) \oplus \mathfrak{s p i n}(r)$ & $\mathfrak{s p}\left(m_{1}\right) \oplus \mathfrak{s p}\left(m_{2}\right)$ \\
\hline 5 & $d_{r} m$ & $\mathfrak{s p}(m) \oplus \mathfrak{s p i n}(r)$ & $\mathfrak{s p}(m)$ \\
\hline 6 & $d_{r} m$ & $\mathfrak{u}(m) \oplus \mathfrak{s p i n}(r)$ & $\mathfrak{u}(m)$ \\
\hline 7 & $d_{r} m$ & $\mathfrak{s o}(m) \oplus \mathfrak{s p i n}(r)$ & $\mathfrak{s o}(m)$ \\
\hline
\end{tabular}


Proposition 3.1. Let $M$ be an $N$-dimensional almost even-Clifford hermitian manifold. Then

TABLE 3

\begin{tabular}{|c|c|c|}
\hline$r(\bmod 8)$ & $N=\operatorname{dim}(M)$ & upper bound $d_{\text {max }} \geq \operatorname{dim}(\operatorname{Aut}(M))$ \\
\hline 0 & $d_{r}\left(m_{1}+m_{2}\right)$ & $\left(\begin{array}{c}m_{1} \\
2\end{array}\right)+\left(\begin{array}{c}m_{2} \\
2\end{array}\right)+\left(\begin{array}{c}r \\
2\end{array}\right)+d_{r}\left(m_{1}+m_{2}\right)$ \\
\hline 1,7 & $d_{r} m$ & $\left(\begin{array}{c}m \\
2\end{array}\right)+\left(\begin{array}{c}r \\
2\end{array}\right)+d_{r} m$ \\
\hline 2,6 & $d_{r} m$ & $m^{2}+\left(\begin{array}{c}r \\
2\end{array}\right)+d_{r} m$ \\
\hline 3,5 & $d_{r} m$ & $\left(\begin{array}{c}2 m+1 \\
2\end{array}\right)+\left(\begin{array}{c}r \\
2\end{array}\right)+d_{r} m$ \\
\hline 4 & $d_{r}\left(m_{1}+m_{2}\right)$ & $\left(\begin{array}{c}2 m_{1}+1 \\
2\end{array}\right)+\left(\begin{array}{c}2 m_{2}+1 \\
2\end{array}\right)+\left(\begin{array}{l}r \\
2\end{array}\right)+d_{r}\left(m_{1}+m_{2}\right)$ \\
\hline
\end{tabular}

3.2. Large automorphism group. In this subsection, we determine the spaces that support an automorphism group of maximal dimension and prove a gap in the dimension of the automorphism group.

Proposition 3.2. Let $M$ be an $N$-dimensional, rank $r \geq 3$ almost even-Clifford hermitian manifold and assume that the dimension of its automorphism group is maximal. Then, for any $p \in M$, the isotropy subgroup $A_{p}$ of $p$ is conjugate to $C_{S O(N)}(\operatorname{Spin}(r)) \cdot \operatorname{Spin}(r) \subset S O(N)$.

Proof. Let $p \in M$ and let $A_{p}$ denote its isotropy group. The dimension of the orbit of $p$ under $\operatorname{Aut}(M)$ satisfies

$$
\operatorname{dim}(\operatorname{Aut}(M))-\operatorname{dim}\left(A_{p}\right) \leq N
$$

so that

$$
\begin{aligned}
\operatorname{dim}\left(A_{p}\right) & \geq \operatorname{dim}(\operatorname{Aut}(M))-N \\
& =d_{\text {max }}-N \\
& =\operatorname{dim}\left(C_{S O(N)}(\operatorname{Spin}(r)) \cdot \operatorname{Spin}(r)\right) .
\end{aligned}
$$

The Lie algebra $\mathfrak{a}_{p}$ of $A_{p}$ maps injectively into $C_{\mathfrak{s o}(N)}(\widehat{\mathfrak{s p i n}(r)}) \oplus \widehat{\mathfrak{s p i n}(r)}$ since a Killing vector field $X$ is determined by its values $X_{p}$ and $(\nabla X)_{p}$. Hence,

$$
\mathfrak{a}_{p} \cong C_{\mathfrak{s o}(N)}(\widehat{\mathfrak{s p i n}(r)}) \oplus \widehat{\mathfrak{s p i n}(r)} .
$$

Proposition 3.3. Let $M$ be a rank $r \geq 3$ almost even-Clifford hermitian manifold and assume that the dimension of its automorphism group is maximal. Then, $M$ is symmetric and the almost even-Clifford hermitian structure is parallel.

Proof. Let $p \in M$ and $A_{p}$ denote its isotropy group. We know that $A_{p}=$ $C_{S O(N)}(\operatorname{Spin}(r)) \cdot \operatorname{Spin}(r)$. Since $C_{S O(N)}(\operatorname{Spin}(r))$ contains 1 and $\operatorname{Spin}(r)$ contains -1 , we have $-1 \in A_{p}$. Thus, there is an element $g \in A_{p}$ whose derivative $d g_{p}=-\mathrm{Id}_{T_{p} M}$ is in the image of the isotropy representation of $A_{p}$ on $T_{p} M$. In other words, the automorphism $g$ is a (global) symmetry at $p$ and $M$ is symmetric. Since these symmetries generate the translations along geodesics, $M$ has a transitive group of automorphisms, not just isometries. 
Proceeding as in p. 264 of [5], given a vector field $W$ with $W_{p} \neq 0$, let $c(t)$ be the geodesic with $\dot{c}(0)=W_{p}$ and $\tau_{t}$ be the group of translations along $c$. Then

$$
Z_{q}:=\frac{d}{d t} \tau_{t}(q)_{\mid t=0}
$$

is an infitesimal automorphism since $\tau_{t}$ are automorphisms. We have that $W_{p}=Z_{p}$. For $v \in T_{p} M$, let $\gamma(s)$ be a curve with $\gamma^{\prime}(0)=v$. Then

$$
\begin{aligned}
\nabla_{v} Z_{p} & =\nabla_{\frac{\partial}{\partial s}} \frac{\partial}{\partial t} \tau_{t}(\gamma(s))_{\mid s=t=0} \\
& =\nabla_{\frac{\partial}{\partial t}} \frac{\partial}{\partial s} \tau_{t}(\gamma(s))_{\mid s=t=0} \\
& =\nabla_{\frac{\partial}{\partial t}} D \tau_{t}(v)_{\mid t=0} \\
& =0
\end{aligned}
$$

since $D_{\tau_{t}}$ is parallel transport along $c$ and $D_{\tau_{t}}(v)$ is a parallel vector field along $c$. Hence, for any vector $W_{p} \in T_{p} M$, we have an infinitesimal automorphism $Z$ such that

$$
Z_{p}=W_{p} \quad \text { and } \quad(\nabla Z)_{p}=0
$$

Now, recall that

$$
\nabla_{Z}(Y)=\mathcal{L}_{Z} Y+\nabla_{Y} Z
$$

On the one hand,

$$
\begin{aligned}
\left(\nabla_{W}\left(J_{i j}(Y)\right)\right)_{p} & =\left(\nabla_{Z}\left(J_{i j}(Y)\right)\right)_{p} \\
& =\left(\left(\nabla_{Z} J_{i j}\right)(Y)\right)_{p}+\left(J_{i j}\left(\nabla_{Z} Y\right)\right)_{p}
\end{aligned}
$$

and, on the other hand,

$$
\begin{aligned}
\left(\mathcal{L}_{Z}\left(J_{i j}(Y)\right)+\nabla_{J_{i j}(Y)} Z\right)_{p}= & \left(\left(\mathcal{L}_{Z} J_{i j}\right)(Y)\right)_{p}+\left(J_{i j}\left(\mathcal{L}_{Z} Y\right)\right)_{p}+\left(\nabla_{J_{i j}(Y)} Z\right)_{p} \\
= & \left(\sum_{k<l} \alpha_{k l}^{(i j)} J_{k l}(Y)\right)_{p}+\left(J_{i j}\left(\nabla_{Z} Y\right)\right)_{p} \\
& -\left(J_{i j}\left(\nabla_{Y} Z\right)\right)_{p}+\left(\nabla_{J_{i j}(Y)} Z\right)_{p}
\end{aligned}
$$

so that

$$
\begin{aligned}
\left(\left(\nabla_{Z} J_{i j}\right)(Y)\right)_{p} & =\left(\sum_{k<l} \alpha_{k l}^{(i j)} J_{k l}(Y)\right)_{p}-\left(J_{i j}\left(\nabla_{Y} Z\right)\right)_{p}+\left(\nabla_{J_{i j}(Y)} Z\right)_{p} \\
& =\left(\sum_{k<l} \alpha_{k l}^{(i j)} J_{k l}(Y)\right)_{p}+\left[(\nabla Z)_{p},\left(J_{i j}\right)_{p}\right]\left(Y_{p}\right) \\
& =\left(\sum_{k<l} \alpha_{k l}^{(i j)} J_{k l}(Y)\right)_{p},
\end{aligned}
$$


i.e.

$$
\left(\nabla_{W} J_{i j}\right)_{p}=\left(\sum_{k<l} \alpha_{k l}^{(i j)} J_{k l}\right)_{p}
$$

Theorem 3.1. Let $M$ be a simply connected Riemannian almost even-Clifford hermitian manifold of rank $r \geq 3$ such that the dimension of its group of automorphisms is maximal. Then $M$ is isometric to one of the following spaces:

TABLE 4

\begin{tabular}{|c|c|}
\hline$r$ & $M$ \\
\hline arbitrary & $\tilde{\Delta}_{r}^{\times m}$ or $\left(\tilde{\Delta}_{r}^{+}\right)^{\times m_{1}} \oplus\left(\tilde{\Delta}_{r}^{-}\right)^{\times m_{2}}$, for some $m, m_{1}, m_{2} \in \mathbb{N}$ \\
\hline 3 & $S p(k+1) /(S p(k) \times S p(1)), S p(k, 1) /(S p(k) \times S p(1))$ \\
\hline 4 & $M_{1} \times M_{2}$, where $M_{i}=\left(\tilde{\Delta}_{3}\right)^{\times m}, S p(k+1) /(S p(k) \times S p(1))$, \\
& $S p(k, 1) /(S p(k) \times S p(1))$ \\
\hline 5 & $S p(k+2) /(S p(k) \times S p(2)), S p(k, 2) /(S p(k) \times S p(2))$ \\
\hline 6 & $S U(k+4) / S(U(k) \times U(4)), S U(k, 4) / S(U(k) \times U(4))$ \\
\hline 8 & $S O(k+8) /(S O(k) \times S O(8)), S O(k, 8) /(S O(k) \times S O(8))$ \\
\hline 9 & $F_{4} / S_{p i n}(9), F_{4}^{-20} / \operatorname{Spin}(9)$ \\
\hline 10 & $E_{6} /(\operatorname{Spin}(10) \cdot U(1)), E_{6}^{-14} /(\operatorname{Spin}(10) \cdot U(1))$ \\
\hline 12 & $E_{7} /(\operatorname{Spin}(12) \cdot S U(2)), E_{7}^{-5} /\left(\operatorname{Spin}_{1}(12) \cdot S U(2)\right)$ \\
\hline 16 & $E_{8} / \operatorname{Spin}^{+}(16), E_{8}^{8} / \operatorname{Spin}^{+}(16)$ \\
\hline
\end{tabular}

Proof. The flat case is clear and the case $r=3$ was dealt with in [11.

For $r=4$, by [9], $M$ is a Riemannian product $M_{1} \times M_{2}$ of quaternion-Kähler manifolds. We claim that

$$
\operatorname{dim}(\operatorname{Aut}(M))=\operatorname{dim}\left(\operatorname{Aut}\left(M_{1}\right)\right)+\operatorname{dim}\left(\operatorname{Aut}\left(M_{2}\right)\right)
$$

Indeed, let $X \in \operatorname{aut}(M)$ be an infinitesimal automorphism of $M, X=X_{1}+X_{2}$ with $X_{1} \in \Gamma\left(T M_{1}\right)$ and $X_{2} \in \Gamma\left(T M_{2}\right)$. We will prove that $X_{1} \in \operatorname{aut}\left(M_{1}\right)$ and $X_{2} \in \operatorname{aut}\left(M_{2}\right)$. First note that $X_{1}$ and $X_{2}$ are Killing vector fields.

Recall that

$$
\mathcal{L}_{X} J_{i j}=\sum_{k<l} \alpha_{k l}^{(i j)} J_{k l}
$$

as well as the endomorphisms 9

$$
J_{12}^{ \pm}= \pm \frac{1}{2}\left(J_{14} \pm J_{23}\right), \quad J_{31}^{ \pm}= \pm \frac{1}{2}\left(J_{13} \mp J_{24}\right), \quad J_{23}^{ \pm}= \pm \frac{1}{2}\left(J_{12} \pm J_{34}\right),
$$


where $J_{k l}^{-}$and $J_{k l}^{+}$vanish on $M_{1}$ and $M_{2}$ respectively. Let $Z=Z_{1}+Z_{2}$ with $Z_{1} \in \Gamma\left(T M_{1}\right)$ and $Z_{2} \in \Gamma\left(T M_{2}\right)$,

$$
\begin{aligned}
& \left(\mathcal{L}_{X_{1}} J_{i j}^{+}\right)\left(Z_{1}\right)_{p}=\mathcal{L}_{X_{1}}\left(J_{i j}^{+}\left(Z_{1}\right)\right)_{p}-J_{i j}^{+}\left(\mathcal{L}_{X_{1}} Z_{1}\right)_{p} \in T_{p} M_{1}, \\
& \left(\mathcal{L}_{X_{1}} J_{i j}^{+}\right)\left(Z_{2}\right)_{p}=\mathcal{L}_{X_{1}}\left(J_{i j}^{+}\left(Z_{2}\right)\right)_{p}-J_{i j}^{+}\left(\mathcal{L}_{X_{1}} Z_{2}\right)_{p}=0, \\
& \left(\mathcal{L}_{X_{2}} J_{i j}^{+}\right)\left(Z_{1}\right)_{p}=\mathcal{L}_{X_{2}}\left(J_{i j}^{+}\left(Z_{1}\right)\right)_{p}-J_{i j}^{+}\left(\mathcal{L}_{X_{2}} Z_{1}\right)_{p}=0, \\
& \left(\mathcal{L}_{X_{2}} J_{i j}^{+}\right)\left(Z_{2}\right)_{p}=\mathcal{L}_{X_{2}}\left(J_{i j}^{+}\left(Z_{2}\right)\right)_{p}-J_{i j}^{+}\left(\mathcal{L}_{X_{2}} Z_{2}\right)_{p}=0,
\end{aligned}
$$

i.e. $\mathcal{L}_{X} J_{i j}^{+}=\mathcal{L}_{X_{1}} J_{i j}^{+} \in \operatorname{End}\left(T M_{1}\right)$. Similarly, $\mathcal{L}_{X} J_{i j}^{-}=\mathcal{L}_{X_{2}} J_{i j}^{-} \in \operatorname{End}\left(T M_{2}\right)$. Now consider, for instance,

$$
\begin{aligned}
\mathcal{L}_{X} J_{12}^{+} & =\frac{1}{2} \mathcal{L}_{X}\left(J_{14}+J_{23}\right) \\
& =\frac{1}{2} \sum_{k<l} \alpha_{k l}^{(14)} J_{k l}+\sum_{k<l} \alpha_{k l}^{(23)} J_{k l} \\
& =\frac{1}{2} \sum \beta_{s t} J_{s t}^{+}+\sum \gamma_{s t} J_{s t}^{-},
\end{aligned}
$$

for some functions $\beta_{s t}$ and $\gamma_{s t}$. Since $\mathcal{L}_{X} J_{12}^{+} \in \operatorname{End}\left(T M_{1}\right)$, all the coefficients $\gamma_{s t}=0$. Therefore

$$
\mathcal{L}_{X_{1}} J_{12}^{+}=\sum \beta_{s t} J_{s t}^{+} .
$$

By similar calculations $\mathcal{L}_{X_{1}} J_{i j}^{+}=\sum \beta_{s t}^{(i j)} J_{s t}^{+}$and $\mathcal{L}_{X_{2}} J_{i j}^{-}=\sum \gamma_{s t}^{(i j)} J_{s t}^{-}$, i.e. $X_{1} \in$ $\operatorname{aut}\left(M_{1}\right)$ and $X_{2} \in \operatorname{aut}\left(M_{2}\right)$. Now let $m_{i}=\operatorname{dim}\left(M_{i}\right) / 4, i=1,2$. Since

$$
\begin{aligned}
\operatorname{dim}(\operatorname{Aut}(M)) & =\left(\begin{array}{c}
2 m_{1}+1 \\
2
\end{array}\right)+\left(\begin{array}{c}
2 m_{2}+1 \\
2
\end{array}\right)+6+4 m_{1}+4 m_{2} \\
& =\operatorname{dim}\left(\operatorname{Aut}\left(M_{1}\right)\right)+\operatorname{dim}\left(\operatorname{Aut}\left(M_{2}\right)\right), \\
\operatorname{dim}\left(\operatorname{Aut}\left(M_{i}\right)\right) & \leq\left(\begin{array}{c}
2 m_{i}+1 \\
2
\end{array}\right)+3+4 m_{i},
\end{aligned}
$$

we must have

$$
\operatorname{dim}\left(\operatorname{Aut}\left(M_{i}\right)\right)=\left(\begin{array}{c}
2 m_{i}+1 \\
2
\end{array}\right)+3+4 m_{i} .
$$

Therefore, the dimensions of the automorphism groups of $M_{1}$ and $M_{2}$ are maximal.

For $r \geq 5$, the spaces carrying a parallel even Clifford structure were classified in [9]. They are either locally symmetric or 8-dimensional. For the 8-dimensional case, they should have rank $5,6,7$ or 8 . In [9], they proved that this is equivalent to being quaternion-Kahler, Kahler, with holonomy contained in Spin(7), or Riemannian respectively. Our bounds coincide with the maximal dimension of each of these types in rank 5, 6 and 8. Therefore, for $r=5$ we only have the quaternionic Euclidean space $\mathbb{H}^{2}$, the quaternionic projective space $\mathbb{H}^{2}$, or the quaternionic hyperbolic space. For $r=6$ we have $\mathbb{C}^{2}$, the open ball with Kähler structure with negative constant holomorphic sectional curvature, or the complex projective space $\mathbb{C P}^{2}$. For $r=8$ we have $\mathbb{R}^{8}, S^{8}$ or $\mathbb{R P}^{8}$. For $r=7$ our bound is the maximal one for a Riemannian manifold. The symmetric spaces carrying parallel even Clifford strucutures are listed in Table 4 . We claim that the dimension of the automorphism group of each space listed is maximal. Indeed, let $M=G / K$ be one of the spaces in Table 4, where $G$ is the group of isometries of $M$. We need to prove that every 
Killing vector is an infinitesimal automorphism of $M$. If $X \in \mathfrak{g}$ is a Killing vector field,

$$
\begin{aligned}
\left(\mathcal{L}_{X} J_{i j}\right)(Z) & =\mathcal{L}_{X}\left(J_{i j}(Z)\right)-J_{i j}\left(\mathcal{L}_{X} Z\right) \\
& =\nabla_{X}\left(J_{i j}(Z)\right)-\nabla_{J_{i j}(Z)} X-J_{i j}\left(\nabla_{X} Z\right)+J_{i j}\left(\nabla_{Z} X\right) \\
& =\left(\nabla_{X} J_{i j}\right)(Z)-\left[\nabla X, J_{i j}\right](Z) \\
& =\left(\sum_{k<l} a_{k l}^{(i j)} J_{k l}\right)(Z)-\left[\nabla X, J_{i j}\right](Z)
\end{aligned}
$$

since the almost even-Clifford hermitian structure is parallel.

Recall that

and at a point $p \in M$,

$$
\mathfrak{g}=\mathfrak{k}+\mathfrak{m},
$$

$$
\mathfrak{m} \cong T_{p} M,
$$

and from the list of possible spaces

$$
\mathfrak{k} \cong \mathfrak{a}_{p} \cong C_{\mathfrak{s o}(N)}(\widehat{\mathfrak{s p i n}(r)}) \oplus \widehat{\mathfrak{s p i n}(r)} .
$$

On the other hand, since $M$ is symmetric,

$$
\mathfrak{g} \cong \mathfrak{b}_{p} \oplus T_{p} M
$$

so that $\left.\mathfrak{b}_{p} \cong \mathfrak{k} \cong C_{\mathfrak{s o}(N)}(\widehat{\mathfrak{s p i n}(r)}) \oplus \widehat{\mathfrak{s p i n}(r)}\right)$ and

$$
\left[\nabla X, J_{i j}\right]=\sum_{k<l} b_{k l}^{(i j)} J_{k l}
$$

i.e.

$$
\mathcal{L}_{X} J_{i j}=\sum_{k<l}\left(a_{k l}^{(i j)}+b_{k l}^{(i j)}\right) J_{k l}
$$

Theorem 3.2. Let $M$ be an $N$-dimensional rank $r \geq 3$, almost even-Clifford hermitian manifold and $p \in M$. Assume the following constraints:

TABLE 5

\begin{tabular}{|c|c|c|c|}
\hline$r(\bmod 8)$ & $N$ & constraint & extra constraint \\
\hline 0 & $d_{r}\left(m_{1}+m_{2}\right)$ & $\begin{array}{c}m_{1} \geq m_{2}>\left(\begin{array}{l}r \\
2\end{array}\right)+1 \\
\text { or } m_{2} \geq m_{1}>\left(\begin{array}{l}r \\
2\end{array}\right)+1\end{array}$ & $m_{1} \equiv m_{2} \equiv 0(\bmod 2)$ \\
\hline 1,7 & $d_{r} m$ & $m>\left(\begin{array}{c}r \\
2\end{array}\right)+1$ & $m \equiv 0(\bmod 2)$ \\
\hline 2,6 & $d_{r} m$ & $m>\frac{1}{2}\left(\begin{array}{l}r \\
2\end{array}\right)+\frac{1}{2}$ & $m \equiv 0(\bmod 2)$ \\
\hline 3,5 & $d_{r} m$ & $m>\frac{1}{4}\left(\begin{array}{l}r \\
2\end{array}\right)+1$ & \\
\hline 4 & $d_{r}\left(m_{1}+m_{2}\right)$ & $\begin{array}{c}m_{1} \geq m_{2}>\frac{1}{4}\left(\begin{array}{l}r \\
2\end{array}\right)+1 \\
\text { or } m_{2} \geq m_{1}>\frac{1}{4}\left(\begin{array}{l}r \\
2\end{array}\right)+1\end{array}$ & \\
\hline
\end{tabular}

If $\operatorname{dim}(\operatorname{Aut}(M))$ is not maximal, then

$$
\operatorname{dim}(\operatorname{Aut}(M))<d_{\text {max }}-\left(\begin{array}{l}
r \\
2
\end{array}\right) .
$$


Proof. Suppose that

$$
d_{\max }-\left(\begin{array}{l}
r \\
2
\end{array}\right) \leq \operatorname{dim}(\operatorname{Aut}(M))<d_{\max } .
$$

Again, at a point $p \in M$, the isotropy group satisfies

$$
\operatorname{dim}(\operatorname{Aut}(M))-\operatorname{dim}\left(A_{p}\right) \leq N
$$

and

$$
\begin{aligned}
\operatorname{dim}\left(A_{p}\right) & \geq \operatorname{dim}(\operatorname{Aut}(M))-N \\
& \geq d_{\text {max }}-\left(\begin{array}{l}
r \\
2
\end{array}\right)-N \\
& \geq d_{C}:=\operatorname{dim}\left(C_{S O(N)}(\operatorname{Spin}(r))\right) .
\end{aligned}
$$

The Lie algebra $\mathfrak{a}_{p}$ of $A_{p}$ maps injectively into $C_{\mathfrak{s o}(N)}(\widehat{\mathfrak{s p i n}(r)}) \oplus \widehat{\mathfrak{s p i n}(r)}$ since a Killing vector field $X$ is determined by its values $X_{p}$ and $(\nabla X)_{p}$. Consider the compositions of this map with the projections to the two factors

$$
\rho_{1}: \mathfrak{a}_{p} \longrightarrow C_{\mathfrak{s o}(N)}(\widehat{\mathfrak{s p i n}(r)}), \quad \rho_{2}: \mathfrak{a}_{p} \longrightarrow \widehat{\mathfrak{s p i n}(r)} .
$$

The subalgebra $\rho_{1}\left(\mathfrak{a}_{p}\right)$ is either equal to $C_{\mathfrak{s o}(N)}(\widehat{\mathfrak{s p i n}(r)})$ or is contained in a proper maximal subalgebra of $C_{\mathfrak{s o}(N)}(\widehat{\mathfrak{s p i n}(r)})$.

If $r \not \equiv 2,6(\bmod 8)$, the maximal dimension $d_{M}$ of a proper maximal subalgebra of $C_{\mathfrak{s o}(N)}(\widehat{\mathfrak{s p i n}(r)})$ is given in Table 6 (see [8]). Thus, due to the constraints on the multiplicities $m, m_{1}, m_{2}$, if $\rho_{1}\left(\mathfrak{a}_{p}\right)$ is contained in a proper subalgebra of $C_{\mathfrak{s o}(N)}(\widehat{\mathfrak{s p i n}(r)})$,

$$
\begin{aligned}
d_{C} & >d_{M}+\left(\begin{array}{l}
r \\
2
\end{array}\right) \\
& \geq \operatorname{dim}\left(\rho_{1}\left(\mathfrak{a}_{p}\right)\right)+\operatorname{dim}\left(\rho_{2}\left(\mathfrak{a}_{p}\right)\right) \\
& \geq \operatorname{dim}\left(\mathfrak{a}_{p}\right) \\
& \geq d_{C},
\end{aligned}
$$

which is a contradiction. Thus

$$
\rho_{1}\left(\mathfrak{a}_{p}\right)=C_{\mathfrak{s o}(N)}(\widehat{\mathfrak{s p i n}(r)})
$$

and

where

$$
\mathfrak{a}_{p} \cong C_{\mathfrak{s o}(N)}(\widehat{\mathfrak{s p i n}(r)}) \oplus \mathfrak{K} \subset \mathfrak{s o}(N),
$$

$$
\mathfrak{K}:=\operatorname{ker}\left(\left.\rho_{1}\right|_{\mathfrak{a}_{p}}\right) \subset \operatorname{ker}\left(\rho_{1}\right)=\mathfrak{s p i n}(r) .
$$

Therefore $A_{p}=C_{S O(N)}(\operatorname{Spin}(r)) \cdot K$, where $K$ is a closed subgroup of $\operatorname{Spin}(r)$. The extra assumptions on $m, m_{1}$ and $m_{2}$ imply $-1 \in C_{S O(N)}(\operatorname{Spin}(r))$ and $-1 \in A_{p}$.

TABLE 6

\begin{tabular}{|c|c|c|c|}
\hline$r(\bmod 8)$ & $N$ & $d_{M}$ & $d_{C}$ \\
\hline 0 & $d_{r}\left(m_{1}+m_{2}\right)$ & $\max \left\{\left(\begin{array}{c}m_{1}-1 \\
2\end{array}\right)+\left(\begin{array}{c}m_{2} \\
2\end{array}\right),\left(\begin{array}{c}m_{1} \\
2\end{array}\right)+\left(\begin{array}{c}m_{2}-1 \\
2\end{array}\right)\right\}$ & $\left(\begin{array}{c}m_{1} \\
2\end{array}\right)+\left(\begin{array}{c}m_{2} \\
2\end{array}\right)$ \\
\hline 1,7 & $d_{r} m$ & $\left(\begin{array}{c}m_{-}-1 \\
2\end{array}\right)$ & $\left(\begin{array}{c}m \\
2\end{array}\right)$ \\
\hline 3,5 & $d_{r} m$ & $\left(\begin{array}{c}2 m-1 \\
2\end{array}\right)+3$ & $\left(\begin{array}{c}2 m_{+}+1 \\
2\end{array}\right)$ \\
\hline 4 & $d_{r}\left(m_{1}+m_{2}\right)$ & $\max \left\{\left(\begin{array}{c}2 m_{1}-1 \\
2\end{array}\right)+3+\left(\begin{array}{c}2 m_{2}+1 \\
2\end{array}\right),\left(\begin{array}{c}2 m_{1}+1 \\
2\end{array}\right)+\left(\begin{array}{c}2 m_{2}-1 \\
2\end{array}\right)+3\right\}$ & $\left(\begin{array}{c}2 m_{1}+1 \\
2\end{array}\right)+\left(\begin{array}{c}2 m_{2}+1 \\
2\end{array}\right)$ \\
\hline
\end{tabular}


Thus, there is an element $g \in A_{p}$ whose derivative $d g_{p}=-\operatorname{Id}_{T_{p} M}$ is in the image of the isotropy representation of $A_{p}$ on $T_{p} M$. In other words, the automorphism $g$ is a (global) symmetry at $p$ and $M$ is symmetric. Since these symmetries generate the translations along geodesics, $M$ has a transitive group of automorphisms, not just isometries. As in the proof of Proposition 3.3, this implies that the almost evenClifford hermitian structure is parallel. By arguments similar to those in the proof of Theorem 3.1, we have $\operatorname{dim}(\operatorname{Aut}(M))=d_{\max }$, which is again a contradiction.

For $r \equiv \pm 2(\bmod 8), \operatorname{dim}\left(A_{p}\right) \geq d_{C}$ will happen if $\mathfrak{s u}(m) \subset \rho_{1}\left(\mathfrak{a}_{p}\right)$. Hence $A_{p}=H \cdot K$, where $H$ is some subgroup of $C_{S O(N)}(\operatorname{Spin}(r))$ containing $S U(m)$ and $K$ is some closed subgroup of $\operatorname{Spin}(r)$. Since we are assuming $m$ is even, $-1 \in S U(m)$ and $-1 \in A_{p}$. Therefore $M$ is symmetric; and again the proofs of Proposition 3.3 and Theorem 3.1 imply $\operatorname{dim}(\operatorname{Aut}(M))=d_{\max }$.

Remark. The constraints in the previous theorem are given in order to ensure that $-1 \in A_{p}$. If we were to relax them or change them, a more detailed analysis of the possible subgroups $K \in \operatorname{Spin}(r)$ would be needed.

\section{ACKNOWLEDGMEnTs}

The second named author would like to thank the International Centre for Theoretical Physics and the Institut des Hautes Études Scientifiques for their hospitality and support.

\section{RefERENCES}

[1] Gerardo Arizmendi and Rafael Herrera, Centralizers of spin subalgebras, J. Geom. Phys. 97 (2015), 77-92, DOI 10.1016/j.geomphys.2015.07.002. MR3385118

[2] Thomas Friedrich, Dirac operators in Riemannian geometry, Graduate Studies in Mathematics, vol. 25, American Mathematical Society, Providence, RI, 2000. Translated from the 1997 German original by Andreas Nestke. MR1777332 (2001c:58017)

[3] Alfred Gray and Luis M. Hervella, The sixteen classes of almost Hermitian manifolds and their linear invariants, Ann. Mat. Pura Appl. (4) 123 (1980), 35-58, DOI 10.1007/BF01796539. MR581924 (81m:53045)

[4] Shigeru Ishihara, Groups of isometries of pseudo-Hermitian spaces. I, II, Proc. Japan Acad. 30 (1954), 940-945. MR0071096 (17,85b) II 31 (1955), 418-420. MR0076411 (17,895b)

[5] Jürgen Jost, Riemannian geometry and geometric analysis, 5th ed., Universitext, SpringerVerlag, Berlin, 2008. MR2431897

[6] Shoshichi Kobayashi and Katsumi Nomizu, Foundations of differential geometry. Vol I, Interscience Publishers, a division of John Wiley \& Sons, New York-London, 1963. MR.0152974 (27 \#2945)

[7] H. Blaine Lawson Jr. and Marie-Louise Michelsohn, Spin geometry, Princeton Mathematical Series, vol. 38, Princeton University Press, Princeton, NJ, 1989. MR1031992 (91g:53001)

[8] L. N. Mann, Gaps in the dimensions of transformation groups, Illinois J. Math. 10 (1966), 532-546. MR0200387 (34 \#282)

[9] Andrei Moroianu and Uwe Semmelmann, Clifford structure on Riemannian manifolds, Adv. Math. 228 (2011), no. 2, 940-967, DOI 10.1016/j.aim.2011.06.006. MR2822214(2012g:53083)

[10] Andrei Moroianu and Mihaela Pilca, Higher rank homogeneous Clifford structures, J. Lond. Math. Soc. (2) 87 (2013), no. 2, 384-400, DOI 10.1112/jlms/jds061. MR3046277

[11] Noemi Santana, Almost quaternion-Hermitian manifolds with large automorphism group, J. Geom. Phys. 61 (2011), no. 1, 347-351, DOI 10.1016/j.geomphys.2010.10.008. MR2747006 (2012b:53081)

[12] S. Tanno, The automorphism groups of almost Hermitian manifolds, Kodai Math. Sem. Rep. Volume 25, 2, 1973. 
[13] Hsien-Chung Wang, On Finsler spaces with completely integrable equations of Killing, J. London Math. Soc. 22 (1947), 5-9. MR.0022431(9,206h)

[14] Hidekiyo Wakakuwa, On n-dimensional Riemannian spaces admitting some groups of motions of order less than $n(n-1) / 2$, Tôhoku Math. J. (2) 6 (1954), 121-134. MR0068885 $(16,956 \mathrm{~b})$

Centro de Investigación en Matemáticas, A. P. 402, Guanajuato, Gto., C.P. 36000, MÉxiCO.

E-mail address: gerardo@cimat.mx

Centro de Investigación en Matemáticas, A. P. 402, Guanajuato, Gto., C.P. 36000, MÉXICO

E-mail address: rherrera@cimat.mx

Instituto de Matemáticas, UnAm, Unidad Cuernavaca, A.P. 6-60, C.P. 62131, CuerNAVACA, Morelos, MÉxico.

E-mail address: noemi.santana@im.unam.mx 